Pacific

Journal of

Mathematics

\title{
VECTOR FIELDS, TORUS ACTIONS AND EQUIVARIANT COHOMOLOGY
}

Jim CARrell, Kiumars KaVeh AND Volker PupPe 


\title{
VECTOR FIELDS, TORUS ACTIONS AND EQUIVARIANT COHOMOLOGY
}

\author{
Jim Carrell, Kiumars Kaveh And Volker Puppe
}

\begin{abstract}
We give an explicit connection between the holomorphic equivariant cohomology as defined by Carrell and Lieberman and the usual equivariant cohomology of Borel and Cartan.

Let $X$ be a smooth complex projective variety equipped with a $\mathbb{C}^{*}$-action with fixed point set $Z$. By results of Carrell and Lieberman, there exists a filtration $F_{0} \subset F_{1} \subset \cdots$ of $H^{*}(Z, \mathbb{C})$ such that $\operatorname{Gr} H^{*}(Z, \mathbb{C}) \cong H^{*}(X, \mathbb{C})$ as graded algebras. We give here an explicit connection between this filtration and the $\mathbb{C}^{*}$-equivariant cohomology of $X$.
\end{abstract}

\section{Introduction}

Let $X$ denote a compact Kähler manifold, and suppose $V$ denotes a holomorphic vector field on $X$ whose zero set $Z$ is nonempty. Let $\Omega_{X}^{p}$ denote the sheaf of holomorphic $p$-forms on $X$. The contraction operator $i_{V}$ defines a complex of sheaves

$$
0 \rightarrow \Omega_{X}^{n} \rightarrow \Omega_{X}^{n-1} \rightarrow \cdots \rightarrow \Omega_{X}^{1} \rightarrow \Omega_{X}^{0} \rightarrow 0,
$$

where $n=\operatorname{dim} X$, and an old result of the first author and David Lieberman [1973; 1977] states that the spectral sequence associated to this complex degenerates at its $E_{1}$ term, namely $H^{*}\left(X, \Omega^{*}\right)$ (see Section 2 for a review of this result). This fact, which uses the Deligne Degeneracy Criterion, implies the vanishing statement

$$
H^{p}\left(X, \Omega^{q}\right)=0 \quad \text { if }|p-q|<\operatorname{dim} Z,
$$

and yields a description of the Dolbeault cohomology algebra $H^{*}\left(X, \Omega_{X}^{*}\right)$ of $X$ as the graded $\mathbb{C}$-algebra associated to the filtration of the hypercohomology $H^{*}\left(K_{X}\right)$, which is a ring since $i_{V}$ is a derivation. Although this result has enabled descriptions of cohomology in a number of special cases, for example, algebraic homogeneous spaces [Akyıldız 1982; Carrell 1992], Schubert varieties [Carrell 1992] and toric varieties [Kaveh 2005], the proof itself in [Carrell and Lieberman 1977] doesn't give any insight into how the filtration can be described. This problem is the motivation of the present paper. In fact, we will show that equivariant cohomology

MSC2000: primary 14F43; secondary 57R91.

Keywords: equivariant cohomology, holomorphic vector field, equivariant vector field, torus action. 
and localization give a more transparent way of approaching the theory, provided that $V$ is generated by a $\mathbb{C}^{*}$-action, which also solves the filtration question.

Throughout the paper, $V$ will denote a holomorphic vector field generated by a $\mathbb{C}^{*}$-action. The only assumption on the fixed point set $Z$ of this action is that it be nonempty. It is well known that $Z$ is also a smooth Kähler subvariety. Let $H^{p, q}(X)=H^{q}\left(X, \Omega^{p}\right)$, and recall the Hodge decomposition of the cohomology algebra of $X$ :

Also, for each $s \in \mathbb{Z}$, put

$$
H^{*}(X, \mathbb{C})=\bigoplus_{p+q=*} H^{p, q}(X) .
$$

$$
\mathscr{H}^{s}(X)=\bigoplus_{q-p=s} H^{p, q}(X)
$$

Then $\mathscr{H}^{*}(X)$ is a graded $\mathbb{C}$-algebra. Note that $H^{*}(X, \mathbb{C})=\bigoplus_{s} \mathscr{H}^{s}(X)$ (but not as graded algebras). The following result summarizes what is known in this setting.

Theorem 1.1. Let $X$ be compact Kähler and admit a $\mathbb{C}^{*}$-action with a nonempty fixed point set $Z$. Let $V$ be the holomorphic vector field on $X$ determined by this action and $K_{X}^{*}$ the hypercohomology determined by the spectral sequence associated to $V$. Then, for all $s \in \mathbb{Z}$ :

(i) $\operatorname{dim} H^{s}\left(K_{X}^{*}\right)=\operatorname{dim} \mathscr{H}^{s}(X)$;

(ii) there exists $a \mathbb{C}$-algebra isomorphism

$$
H^{s}\left(K_{X}^{*}\right) \cong \mathscr{H}^{s}(Z)
$$

(iii) we have

$$
\sum_{q-p=s} \operatorname{dim} H^{p, q}(X)=\sum_{q-p=s} \operatorname{dim} H^{p, q}(Z)
$$

(iv) there exists a filtration of $\mathscr{H}^{*}(Z)$ that yields an isomorphism of graded rings

$$
\bigoplus_{s} \mathscr{H}^{s}(X) \cong \operatorname{Gr} \mathscr{H}^{*}(Z) \text {. }
$$

In the above, (i) follows from the degeneracy of the spectral sequence of $V$. The isomorphism (ii) is proven in [Carrell and Sommese 1979], and (iii) follows from the first two parts. The last is in fact treated in several papers, for example, [Carrell and Sommese 1979; Fujiki 1979; Ginzburg 1987]. Also see [Feng 2003] for a proof that doesn't use $\mathbb{C}^{*}$-actions but assumes $V$ vanishes transversely along $Z$.

Spaces admitting a $\mathbb{C}^{*}$-action often have the property that $H^{p, q}(X)=0$ if $p \neq q$ (for example, algebraic homogeneous spaces, projective toric varieties and, more generally, spherical varieties). For such $X, H^{2 p}(X, \mathbb{C})=H^{p}\left(X, \Omega^{p}\right)$ and $H^{2 p+1}(X, \mathbb{C})=0$ for all $p \geq 0$. By (iii), the same is true for $Z$. Thus, $\mathscr{H}^{s}(X)=H^{2 s}(X, \mathbb{C})$ and similarly $\mathscr{H}^{*}(Z)=H^{*}(Z, \mathbb{C})$. Hence the map in (iv) 
reduces to the graded $\mathbb{C}$-algebra isomorphism

$$
H^{*}(X, \mathbb{C}) \cong \operatorname{Gr} H^{*}(Z, \mathbb{C}) .
$$

We note, however, that the filtration on $H^{*}(Z, \mathbb{C})$ has nothing to do with the natural filtration arising from the usual grading of cohomology.

The plan of the paper is as follows. We will use Section 2 to review the spectral sequence of a holomorphic vector field and Section 3 to recall some basic facts about equivariant cohomology and the Cartan complex. Our main results, Theorems 4.2 and 4.4, are proved in Section 4. Theorem 1.1 follows readily from these two results. In Section 5, we give a simple proof of a result in [Carrell 1995] on regular actions, namely, actions of the $2 \times 2$ upper triangular matrices over $\mathbb{C}$ of determinant one such that the unipotent subgroup has a unique fixed point. The equivariant cohomology of these varieties was described in [Brion and Carrell 2004]. In Section 6 we consider some examples.

A few comments about the proofs in Section 4 are in order. Let $T$ denote the compact torus in $\mathbb{C}^{*}$, and suppose $H_{T}^{*}(X, \mathbb{C})$ denotes the $T$-equivariant cohomology of $X$ over $\mathbb{C}$. One knows $H_{T}^{*}(X, \mathbb{C})$ is a free $\mathbb{C}[t]$-module of rank $H^{*}(X, \mathbb{C})$, so, as a $\mathbb{C}[t]$-module, $H_{T}^{*}(X, \mathbb{C}) \cong \mathbb{C}[t] \otimes H^{*}(X, \mathbb{C})$. Recently, Teleman [2000] and Lillywhite [2003] have defined Dolbeault equivariant cohomology groups $H_{T, \bar{\partial}}^{p, q}(X)$ for $X$ and showed that $H_{T}^{*}(X, \mathbb{C})$ admits the usual Hodge decomposition provided $X$ is compact Kähler. This allows us to define the groups $\mathscr{H}_{T}^{s}(X)$ analogous to the groups $\mathscr{H}^{s}(X)$ defined above. We will show that evaluating polynomials at $t=1$ gives a map (of $\mathbb{C}$-algebras) $\mathscr{H}_{T}^{*}(X, \mathbb{C}) \rightarrow H^{*}\left(K_{X}^{*}\right)$. (This idea is suggested by a paper of the third author [Puppe 1979/80].) The key result Theorem 1.1(ii) follows from localization in equivariant cohomology. The filtration of $H^{*}\left(K_{X}^{*}\right)$ essentially turns out to be the image of a canonical filtration of $\mathscr{H}_{T}^{*}(Z, \mathbb{C}) \rightarrow H^{*}\left(K_{Z}^{*}\right)=\mathscr{H}^{*}(Z)$ via the above "strange" map.

\section{Zeros of holomorphic vector fields and cohomology}

The purpose of this section is to review the spectral sequence associated to a holomorphic vector field [Carrell and Lieberman 1973; 1977]. Let $X$ denote a connected compact Kähler manifold of dimension $n$ with sheaf of holomorphic functions $\mathscr{O}_{X}$ and sheaves $\Omega_{X}^{p}$ of holomorphic $p$-forms for $p>0$. The contraction operator $i_{V}: \Omega_{X}^{p} \rightarrow \Omega_{X}^{p-1}$ defines the Koszul complex

$$
0 \rightarrow \Omega_{X}^{n} \rightarrow \Omega_{X}^{n-1} \rightarrow \cdots \rightarrow \Omega_{X}^{1} \rightarrow \mathrm{O}_{X} \rightarrow 0 .
$$

In addition, for all $\phi, \omega \in \Omega_{X}^{*}$,

$$
i_{V}(\phi \wedge \omega)=i_{V} \phi \wedge \omega+(-1)^{p} \phi \wedge i_{V} \omega
$$


if $\phi \in \Omega_{X}^{p}$. Let $A^{p, q}(X)$ denote the smooth forms on $X$ of type $(p, q)$. The $\bar{\partial}$ operator $A^{p, q} \rightarrow A^{p, q+1}$ anticommutes with $i_{V}$, so $\left(\bar{\partial}-i_{V}\right)^{2}=0$. Put

$$
K_{X}^{s}=\bigoplus_{q-p=s} A^{p, q},
$$

and define $D: K_{X}^{s} \rightarrow K_{X}^{s+1}$ to be $\bar{\partial}-i_{V}$. Then because $D^{2}=0$, we obtain cohomology groups $H^{s}\left(K_{X}^{*}\right)$. Moreover, $K_{X}^{*}$ is a differential graded algebra under the exterior product, so the cohomology groups form a graded $\mathbb{C}$-algebra $H^{*}\left(K_{X}^{*}\right)$. Let $F_{\bullet}=F_{0} \subset F_{1} \subset F_{2} \subset \cdots \subset F_{n}$ be the filtration of the double complex $A^{*, *}(X)$, with $F_{i}=\bigoplus_{r \leq i} A^{r, *}(X)$. Since $i_{V}$ is a derivation, we obtain filtrations $F_{\bullet} H^{s}\left(K_{X}^{*}\right)$ for all $s$ such that

$$
F_{i} H^{s}\left(K_{X}^{*}\right) F_{j} H^{t}\left(K_{X}^{*}\right) \subset F_{i+j} H^{s+t}\left(K_{X}^{*}\right) .
$$

Now consider the spectral sequence

$$
E_{1}^{-p, q}=H^{q}\left(X, \Omega_{X}^{p}\right) \Rightarrow H^{q-p}\left(K_{X}^{*}\right) .
$$

The main result is:

Theorem 2.1 [Carrell and Lieberman 1973; 1977]. If $V$ has zeros, then all differentials in (2) are trivial. Consequently $E_{1}=E_{\infty}$, and there are $\mathbb{C}$-linear isomorphisms

$$
H^{p+s}\left(X, \Omega_{X}^{p}\right) \cong F_{p} H^{s}\left(K_{X}^{*}\right) / F_{p-1} H^{s}\left(K_{X}^{*}\right),
$$

for every $p \geq 0$ and $s$ which give an isomorphism of bigraded $\mathbb{C}$-algebras

$$
\bigoplus_{p, s} H^{p+s}\left(X, \Omega_{X}^{p}\right) \cong \bigoplus_{p, s} F_{p} H^{s}\left(K_{X}^{*}\right) / F_{p-1} H^{s}\left(K_{X}^{*}\right) .
$$

\section{Remarks on equivariant cohomology}

In this section, we will briefly recall the two basic definitions of equivariant cohomology due to Borel and Cartan, and state a recent result of Teleman [2000, Theorem 7.3] and Lillywhite [2003, §5.1] on equivariant Dolbeault cohomology. Suppose $G$ is a compact topological group acting on a space $M$. It is well known that there exists a contractible space $E G$ with a free $G$-action. The quotient $B G=E G / G$ is called the classifying space of $G$. Put

$$
M_{G}=(M \times E G) / G .
$$

The equivariant cohomology of $M$ over $\mathbb{C}$ is defined to be

$$
H_{G}^{*}(M)=H^{*}\left(M_{G}, \mathbb{C}\right) .
$$


If $G$ is a compact torus, say $T$, then $H_{T}^{*}$ (point) $=H^{*}(B T)$ is identified with the polynomial ring $S=\mathbb{C}[\operatorname{Lie}(T)]$, which is graded by assigning degree two to linear forms on $\operatorname{Lie}(T)$. Thus, $H_{T}^{*}(M)$ is an $S$-module (via the natural map $\pi: M_{T} \rightarrow$ $B T$ ), and one has the following fundamental fact:

Theorem 3.1 (Localization Theorem). Suppose the compact torus $T$ acts on a space $M$ which admits an equivariant imbedding into a representation of $T$. Then the kernel as well as the cokernel of the canonical map

$$
i^{*}: H_{T}^{*}(M) \rightarrow H_{T}^{*}\left(M^{T}\right)
$$

induced by the inclusion $i: M^{T} \hookrightarrow M$ are torsion modules over $S$. Thus if $H_{T}^{*}(M)$ is a free module over $S$, then $i^{*}$ is injective. Moreover, $i^{*}$ becomes an isomorphism after inverting elements of a finitely generated multiplicative subset of the polynomial algebra $S$.

If $H_{T}^{*}(M)$ is a free $S$-module, then the action of $T$ on $M$ is said to be equivariantly formal. Equivalently, $M$ is equivariantly formal if the spectral sequence of the fibration $M_{T} \rightarrow B T$ collapses.

Remark 3.2. By a result of Frankel [1959], a $\mathbb{C}^{*}$-action with fixed points on a compact Kähler manifold is equivariantly formal for the compact torus $T=S^{1} \subset \mathbb{C}^{*}$. More generally, by a theorem of Kirwan, every Hamiltonian $T$-action on a compact symplectic manifold is equivariantly formal [1984, Proposition 5.8]. Moreover, the hypotheses of Theorem 3.1 hold in the compact symplectic (in particular, compact Kähler) case. For further examples of equivariantly formal spaces, see [Goresky et al. 1998, §14.1].

To recall Cartan's construction of equivariant cohomology [1951], we will assume the space $M$ is a smooth manifold on which $T$ acts smoothly. Let $\Omega^{*}(M)$ be the De Rham complex of $\mathbb{C}$-valued forms on $M$. Define $\Omega_{T}^{*}(M)$ to be the complex consisting of all the polynomial maps $f: \operatorname{Lie}(T) \rightarrow\left(\Omega^{*}(M)\right)^{T}$. Here the superscript denotes the $T$-invariants. This is equivalent to defining $\Omega_{T}^{*}(M)=\left(\Omega^{*}(M) \otimes_{\mathbb{C}} S\right)^{T}$. In particular

$$
\Omega_{T}^{*}:=\Omega_{T}^{*} \text { (point) }=S^{T}=S .
$$

The grading on $\Omega_{T}^{*}(M)$ is defined by $\operatorname{deg}(f)=n+2 p$, if $x \mapsto f(x)$ is of degree $p$ in $x$ and $f(x) \in \Omega^{n}(M)$. The differential

$$
d_{T}: \Omega_{T}^{*}(M) \rightarrow \Omega_{T}^{*}(M)
$$

of this complex is defined by

$$
\left(d_{T} f\right)(x)=d(f(x))-i_{V_{x}} f(x),
$$

where $i_{V_{x}}$ is the contraction with the vector field $V_{x}$ on $M$ generated by $x \in \operatorname{Lie}(T)$. Then $d_{T} \circ d_{T}=0$ and $d_{T}$ increases the degree in $\Omega^{*}(M)$ by 1 . 
Theorem 3.3 [Cartan 1951]. $H_{T}^{*}(M)$ and $H^{*}\left(\Omega_{T}^{*}(M), d_{T}\right)$ are isomorphic graded $\mathbb{C}$-algebras.

If $M$ is a complex manifold and $T$ acts via holomorphic transformations, a Dolbeault version of $T$-equivariant cohomology is constructed in a similar way. For $x \in \operatorname{Lie}(T)$, let $V_{x}=W_{x}+\overline{W_{x}}$ be the splitting of the generating vector field of $x$ into holomorphic and antiholomorphic components. Imitating the Cartan construction, let $A_{T}^{p, *}(M)$ be the complex of all polynomial maps $f$ from $\operatorname{Lie}(T)$ to $\left(A^{p, *}(M)\right)^{T}$. (Note again that this is the same as defining $A_{T}{ }^{p, *}(M)=\left(A^{p, *}(M) \otimes_{\mathbb{C}} S\right)^{T}$ ). Giving bidegree $(1,1)$ to the generators of $S$ defines a bigrading on the algebra $A_{T}^{*, *}(M)=\bigoplus_{p, q} A_{T}^{p, q}(M)$. Define the differential $\bar{\partial}_{T}$ on $A_{T}^{p, *}(M)$ by

$$
\left(\bar{\partial}_{T} f\right)(x)=\bar{\partial}(f(x))-i_{W_{x}} f(x) .
$$

The $q$-th cohomology of the complex $\left(A_{T}^{p, *}(M), \bar{\partial}_{T}\right)$ is called the $(p, q)$-th equivariant Dolbeault cohomology of $M$. It is denoted by $H_{T}^{p, q}(M)$. Finally, put

$$
H_{T, \bar{\partial}}^{m}(M)=\bigoplus_{p+q=m} H_{T}^{p, q}(M) .
$$

We now state a recent result of Lillywhite [2003] and Teleman [2000].

Theorem 3.4 (Equivariant Hodge Decomposition). If $X$ is a compact Kähler manifold with an equivariantly formal $T$-action by holomorphic transformations, then $H_{T, \bar{\partial}}^{*}(X)$ is a free $S$-module, and there exists an isomorphism

$$
H_{T}^{*}(X) \cong H_{T, \bar{\partial}}^{*}(X)
$$

of graded $\mathbb{C}$-algebras.

Finally, we recall the definition of the equivariant Chern classes of a vector bundle. Let $E$ be a complex vector bundle over the a space $M$ on which $T$ acts, and suppose $E$ has a linear action of $T$ lifting the action of $T$. The projection map $p: E \rightarrow M$ defines a map from $E_{T}=E \times_{T} E T$ to $M_{T}=X \times_{T} E T$. This makes $E_{T}$ a vector bundle over $M_{T}$. The $r$-th equivariant Chern class of $E$, denoted by $c_{r}^{T}(E)$, is defined to be the $r$-th Chern class of $E_{T}$. It is clear that $c_{r}^{T}(E) \in H_{T}^{2 r}(M)$.

Remark 3.5. We will need the following fact in Section 5: suppose $M$ is connected and the action of $T$ on $M$ is trivial. Let $E$ be a line bundle with a $T$-action as above. Let the weight of action of $T$ on each fibre of $E$ be $\omega$. Then

$$
c_{1}^{T}(E)=-\omega+c_{1}(E)
$$

in $H_{T}^{2}(X)=\left(S \otimes H^{*}(X)\right)_{2}$. 


\section{The main results}

Now let $X$ denote a connected compact Kähler manifold of dimension $n$ having a $\mathbb{C}^{*}$ action with nonempty fixed point set $Z$, and let $T$ be the compact torus in $\mathbb{C}^{*}$. Let $V$ be the generating vector field of $1 \in \mathbb{C}=\operatorname{Lie}\left(\mathbb{C}^{*}\right)$, and, as before, let $K_{X}^{*}$ denote the total complex of the Koszul complex of the vector field $V$. It is well known that $X^{T}=Z$. From now on, $S=\mathbb{C}[t]$.

The purpose of this section is to derive the results about the spectral sequence of $V$ (in particular, to prove Theorem 1.1) using Dolbeault $T$-equivariant cohomology and to obtain a new picture of the filtration $F_{\bullet}=F_{0} \subset F_{1} \subset \cdots \subset F_{n}$ of $H^{*}\left(K_{X}^{*}\right)$.

We first define a chain map $\tilde{\Phi}_{X}: A_{T}^{*, *}(X) \rightarrow K_{X}^{*}$. Recall that an element of $A_{T}^{*, *}(X)$ is a polynomial map $f: \mathfrak{t} \rightarrow\left(A^{*, *}(X)\right)^{T}$. By Definition (1), if $f \in$ $A_{T}^{p, q}(X)$, then $f(1) \in K_{X}^{q-p}$. Therefore, put

$$
\tilde{\Phi}(f)=f(1) .
$$

Proposition 4.1. $\tilde{\Phi}$ is a cochain map. That is, for $f \in A_{T}^{*, *}(X)$, we have

$$
\tilde{\Phi}\left(\bar{\partial}_{T} f\right)=D(\tilde{\Phi}(f)) .
$$

Proof. $\tilde{\Phi}\left(\bar{\partial}_{T} f\right)=\tilde{\Phi}\left(\bar{\partial} f(x)-i_{V_{x}} f(x)\right)=\bar{\partial} f(1)-i_{V} f(1)=D(f(1))=D(\tilde{\Phi}(f))$. Here $V_{x}$ and $V$ are the generating vector fields of $x \in \operatorname{Lie}(T)$ and $1 \in \operatorname{Lie}(T)$.

It is now convenient to put $\mathscr{H}_{T}^{s}(X)=\bigoplus_{i} H_{T}^{i, i+s}(X)$. Note that by Theorem 3.4, $H_{T}^{*}(X)=\bigoplus_{s} \mathscr{H}_{T}^{s}(X)$. This gives a new grading on $H_{T}^{*}(X)$ by $S$-submodules. We will denote $H_{T}^{*}(X)$ with this grading by $\mathcal{H}_{T}^{*}(X)$. By the above proposition, $\tilde{\Phi}$ induces a map

$$
\Phi_{X, s}: \mathscr{H}_{T}^{s}(X) \rightarrow H^{s}\left(K_{X}^{*}\right) .
$$

It is not hard to check that the $\Phi_{X, s}$ give a $\mathbb{C}$-algebra homomorphism.

Let $\pi$ denote the natural map $\pi: H_{T}^{p, p+s}(X) \rightarrow H^{p, p+s}(X)$ induced by the inclusion $X \hookrightarrow X_{T}$. By equivariant formality, the ordinary cohomology sequence

$$
0 \rightarrow S^{+} H_{T}^{*}(X) \rightarrow H_{T}^{*}(X) \rightarrow H^{*}(X) \rightarrow 0
$$

is exact (compare [Brion 1998, Section 1]), so by the equivariant Hodge decomposition, $\pi$ is surjective for all $p, s$.

Let $\mathscr{H}^{*}(X)$ denote $H^{*}(X, \mathbb{C})$ and grade it with the decomposition $H^{*}(X)=$ $\bigoplus_{s} \mathscr{H}^{s}(X)$, as defined in Section 2. For any $\mathbb{C}$-vector space $V$ and $a \in \mathbb{C}$, let $V[a]$ denote the $S$-module structure on $V$ where $t$ acts via multiplication by $a$. Note that $\operatorname{dim} V[a]$ is the same for all $a$. By (7), we have another exact sequence of $S$-modules

$$
0 \rightarrow S^{+} \mathscr{H}_{T}^{s}(X) \rightarrow \mathscr{H}_{T}^{s}(X) \rightarrow \mathscr{H}^{s}(X)[0] \rightarrow 0
$$


where $S^{+}$denotes the ideal generated by $t$. Hence

$$
\mathscr{H}^{s}(X)[0] \cong \mathscr{H}_{T}^{s}(X) / S^{+} \mathscr{H}_{T}^{s}(X) \cong \mathscr{H}_{T}^{s}(X) \otimes_{S} \mathbb{C}[0],
$$

and therefore

$$
\operatorname{dim}\left(\mathscr{H}_{T}^{s}(X) \otimes_{S} \mathbb{C}[0]\right)=\operatorname{dim} \mathscr{H}^{s}(X)=\sum_{i} \operatorname{dim} H^{i, i+s}(X),
$$

We now prove the first three assertions of Theorem 1.1. First, notice that the chain map $\Phi_{X, s}$ in (6) induces a map $\hat{\Phi}_{X, s}: \mathscr{H}_{T}^{s}(X) \otimes_{S} \mathbb{C}[1] \rightarrow H^{s}\left(K_{X}^{*}\right)[1]=$ $H^{s}\left(K_{X}^{*}\right)$.

Theorem 4.2. The following statements hold for each integer $s$.

(i) $\hat{\Phi}_{X, s}: \mathscr{H}_{T}^{s}(X) \otimes_{S} \mathbb{C}[1] \rightarrow H^{s}\left(K_{X}^{*}\right)$ is a $\mathbb{C}$-linear isomorphism.

(ii) The inclusion mapping $i_{Z}: Z \rightarrow X$ induces $a \mathbb{C}$-algebra isomorphism

$$
i_{Z}^{*}: H^{s}\left(K_{X}^{*}\right) \cong H^{s}\left(K_{Z}^{*}\right)=\mathscr{H}^{s}(Z) .
$$

(iii) In particular, $\sum_{i} \operatorname{dim} H^{i, i+s}(X)=\sum_{i} \operatorname{dim} H^{i, i+s}(Z)$.

Proof. The Localization Theorem 3.1 implies the map $i_{Z}^{*}$ induces an isomorphism

$$
\mathscr{H}_{T}^{s}(X) \otimes_{S} \mathbb{C}[1] \cong \mathscr{H}_{T}^{s}(Z) \otimes_{S} \mathbb{C}[1] .
$$

Since $\mathscr{H}_{T}^{s}(Z) \otimes_{S} \mathbb{C}[0] \cong \mathscr{H}^{s}(Z)=H^{s}\left(K_{Z}^{*}\right)$, and since $\operatorname{dim}\left(\mathscr{H}_{T}^{s}(X) \otimes_{S} \mathbb{C}[a]\right)$ is the rank of $\mathscr{H}_{T}^{s}(X)$ as a free $S$-module for any $a$, we get an isomorphism $\mathscr{H}_{T}^{s}(X) \otimes_{S}$ $\mathbb{C}[1] \cong H^{s}\left(K_{Z}^{*}\right)$, which is nothing more than $i_{Z}^{*} \hat{\Phi}_{X, s}$. It follows that $\hat{\Phi}_{X, s}$ is injective.

To prove part (i), it remains to show $\hat{\Phi}_{X, s}$ is surjective. It suffices to show that $\Phi_{X, s}$ is. By standard reasoning about the spectral sequence of a double complex, we have an edge map $e_{p, s}: F_{p} H^{s}\left(K_{X}^{*}\right) \rightarrow H^{p, p+s}(X)$ whose kernel contains $F_{p-1} H^{s}\left(K_{X}^{*}\right)$. Let $f(t)=\sum_{i} w_{i} t^{i}$, where each $w_{i} \in A^{p-i, p+s-i}(X)$ represents a class in $H_{T}^{p, p+s}(X)$. By definition, $\Phi_{X, s}(f)=\sum_{i} w_{i}$. Moreover,

$$
\pi(f)=w_{0}=e_{p, s}\left(\sum_{i} w_{i}\right) .
$$

In other words, we get the following commutative diagram.

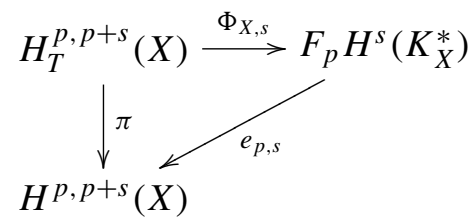

Since $\pi$ is surjective, it follows from this that $\Phi_{X, s}$ is surjective. This concludes the proof of (i). The statements (ii) and (iii) follow immediately. 
Remark 4.3. Theorem 4.2(i) is analogous to the corollary in [Puppe 1974, p. 13]. The proof of Theorem 4.2 implies that the subcomplex of the Koszul complex consisting of $T$-invariant forms is quasi-isomorphic to the Koszul complex itself. By first proving this result directly (which is similar to the well-known result that invariant forms in the deRham complex determine the deRham cohomology) and then using that the equivariant Dolbeault evaluated at $t=1$ is just the invariant Koszul complex, one gets an alternative proof of Theorem 4.2. In this context, the evaluation at $t=1$ is exact, and hence commutes with homology, whereas the evaluation at $t=0$ is not.

Theorem 4.2 realizes two of the goals of the paper: a simple proof that $i_{Z}^{*}$ is a quasiisomorphism, and a proof of the isomorphism (3) of Theorem 2.1 that doesn't use the Deligne Degeneracy Criterion. We note that the isomorphism (4) is a formal consequence of the fact that $i_{V}$ is a derivation.

Let us now comment further on the filtrations. Let $\hat{\Phi}_{X}: \mathscr{H}_{T}^{*}(X) \otimes_{S} \mathbb{C}[1] \rightarrow$ $H^{*}\left(K_{X}^{*}\right)$ be the morphism obtained by combining the $\hat{\Phi}_{X, s}$. Note that $\hat{\Phi}_{X}$ is a $\mathbb{C}$-algebra isomorphism, but not an isomorphism of graded algebras. However, $\mathscr{H}_{T}^{*}(X) \otimes_{S} \mathbb{C}[1]$ and $H^{*}\left(K_{X}^{*}\right)$ are both canonically filtered, the former being the filtration induced from the grading on $\mathscr{H}_{T}^{*}(X)$ and the latter being the filtration introduced in Section 2. More explicitly, if $p \geq 0$, put $F_{p} \mathscr{H}_{T}^{s}(X)=\bigoplus_{i \leq p} H_{T}^{i, i+s}(X)$. If $\Phi_{X, s}$ is the map defined in (6), then, by definition,

$$
\Phi_{X, s}\left(F_{p} \mathscr{H}_{T}^{s}(X)\right) \subset F_{p} H^{s}\left(K_{X}^{*}\right) .
$$

Note that $\Phi_{X, s}$ can be described as the map obtained by composing $\hat{\Phi}_{X, s}$ and the natural map from $\mathscr{H}_{T}^{*}(X)$ to $\mathscr{H}_{T}^{*}(X) \otimes_{S} \mathbb{C}[1]$ sending $\alpha$ to $\alpha \otimes_{S} 1$.

We can now give a geometric description of the filtration of $H^{*}\left(K_{X}^{*}\right)$. Let $\mathscr{R}_{X}$ denote the algebra $\mathscr{H}_{T}^{*}(X) / S^{+} \mathscr{H}_{T}^{*}(X)$. Since the ideal $S^{+} \mathscr{H}_{T}^{*}(X)$ is homogeneous with respect to the grading of $\mathscr{H}_{T}^{*}(X), \mathscr{R}_{X}$ inherits a grading from $\mathscr{H}_{T}^{*}(X)$.

Theorem 4.4. The mapping $\Phi_{X}$ is a surjection of filtered rings. That is, for all s,

$$
\Phi_{X, s}\left(F_{p} \mathscr{H}_{T}^{s}(X)\right)=F_{p} H^{*}\left(K_{X}^{*}\right),
$$

and $\mathscr{R}_{X}$ is isomorphic to both $\mathscr{H}^{*}(X)$ and $\mathrm{Gr} H^{*}\left(K_{X}^{*}\right)$ as graded algebras.

Proof. This follows from (8) and Theorem 4.2(i).

Since the inclusion map $i_{Z}: Z \rightarrow X$ induces a quasi-isomorphism, we immediately obtain a description of the filtration of $H^{*}\left(K_{Z}^{*}\right)$ whose associated graded is $\mathscr{H}^{*}(X)$.

Corollary 4.5. For each $p \geq 0$,

$$
\Phi_{Z} \circ i_{Z}^{*}\left(\bigoplus_{0 \leq i \leq p} H_{T}^{i, i+s}(X)\right)=F_{p} H^{s}\left(K_{Z}^{*}\right) .
$$


We will give an example of how to use this result in the next section. Note also that the natural map

$$
\Delta_{p}: H_{T}^{p, p+s}(X) \rightarrow F_{p} H^{s}\left(K_{X}^{*}\right) \rightarrow H^{p}\left(X, \Omega_{X}^{p+s}\right)
$$

can be described as the $p$-th derivative map

$$
\Delta_{p}(f)=\frac{1}{p !} f^{(p)}(1) .
$$

We now use Theorem 4.2 to prove a vanishing theorem which extends the vanishing result $H^{p, q}(X)=0$ if $|p-q|>\operatorname{dim} Z$.

Theorem 4.6. If $|p-q|>\operatorname{dim} Z$, then $H_{T}^{p, q}(X)=0$.

Proof. Since $H_{T}^{*}(Z)=S \otimes_{\mathbb{C}} H^{*}(Z)$, it follows that

$$
H_{T}^{p, q}(Z)=\bigoplus_{i \leq \min (p, q)} S^{i} \otimes_{\mathbb{C}} H^{p-i, q-i}(Z)
$$

But $|p-q|=|(p-i)-(q-i)|>\operatorname{dim} Z$, so $H_{T}^{p, q}(Z)=0$ as well. By Theorem 3.4, $H_{T}^{p, q}(X) \subset H_{T}^{p+q}(X)$, so the result follows from the Localization Theorem 3.1 since $i^{*}\left(H_{T}^{p, q}(X)\right) \subset H_{T}^{p, q}(Z) \subset H_{T}^{p+q}(Z)$.

\section{An application}

The purpose of this section is to apply our main result to give a simple proof of a fact about the cohomology ring of a regular variety originally proved in [Carrell 1995]. A smooth projective variety $X$ over $\mathbb{C}$ that admits an action of the upper triangular subgroup $\mathfrak{B}$ of $S L_{2}(\mathbb{C})$ whose unipotent radical $\mathfrak{U}$ has a unique fixed point $o$ is said to be regular. Let $\mathfrak{T}$ denote the diagonal torus in $\mathfrak{B}$, and let $T$ be the maximal compact torus in $\mathfrak{T}$. One knows [Carrell 1995] that $X^{\mathfrak{T}}=X^{T}$ is finite, and, moreover, $o \in X^{T}$. In fact, let $\lambda: \mathbb{C}^{*} \rightarrow \mathfrak{T}$ be the isomorphism

$$
t \rightarrow\left(\begin{array}{cc}
t & 0 \\
0 & t^{-1}
\end{array}\right)
$$

Then the Bialynicki-Birula cell $X_{o}=\left\{x \in X \mid \lim _{t \rightarrow \infty} \lambda(t) \cdot x=o\right\}$ is a T-invariant open set in $X$ isomorphic with $\mathbb{C}^{n}$ for $n=\operatorname{dim} X$, and there exist affine coordinates $u_{1}, \ldots, u_{n}$ on $X_{o}$ that are quasihomogeneous of positive degree with respect to the $\mathbb{G}_{m}$-action on $X$ induced by $\lambda$. This grading on $\mathbb{C}\left[X_{o}\right]=\mathbb{C}\left[u_{1}, \ldots, u_{n}\right]$ is called the principal grading. The principal filtration $P_{\bullet}$ of $\mathbb{C}\left[X_{o}\right]$ is given by

$$
P_{i} \mathbb{C}\left[X_{o}\right]=\sum_{j \leq i} \mathbb{C}\left[X_{o}\right]^{j}
$$


where $\mathbb{C}\left[X_{o}\right]^{j}$ denotes the subspace generated by the homogeneous elements of degree $j$. Finally, let

$$
\mu(a)=\left(\begin{array}{ll}
1 & a \\
0 & 1
\end{array}\right)
$$

and let $T_{a}\left(a \in \mathbb{C}^{*}\right)$ be the torus $\mu(a) T \mu(a)^{-1}$. We will now prove the following result.

Theorem 5.1. Suppose $X$ is regular. Then $X^{T_{a}} \subset X_{o}$, so $H^{0}\left(X^{T_{a}}\right)$ is a quotient of $\mathbb{C}\left[X_{o}\right]$ for any $a \in \mathbb{C}^{*}$. Hence it inherits a natural filtration from the principal filtration of $\mathbb{C}\left[X_{o}\right]$, so let $\mathrm{Gr}_{P} H^{0}\left(X^{T_{a}}\right)$ denote the associated graded ring. Then

$$
H^{*}(X) \cong \operatorname{Gr}_{P} H^{0}\left(X^{T_{a}}\right) .
$$

Proof. We will only prove the theorem for $a=1$. The proof for other values of $a$ is similar, after the map $\Phi_{X}$ has been modified. Put $X^{T}=\left\{x_{1}, \ldots, x_{r}\right\}$. Now the diagonal action of $\mathfrak{B}$ on $X \times \mathbb{P}^{1}$ is also regular, with fixed point $(o, 0)$, where 0 represents $[1,0]$ in $\mathbb{P}^{1}$. Let

$$
Z=\bigcup_{i=1}^{r} \overline{\left\{\left(\mu(u) \cdot x_{i}, u^{-1}\right) \mid u \neq 0\right\}} .
$$

Let $\mathscr{L}$ be the reduced intersection $Z \cap\left(X_{o} \times \mathbb{C}\right)$. Clearly, $\mathscr{L}$ is $\mathfrak{T}$-stable, hence its coordinate ring $\mathbb{C}[\mathscr{L}]$ has a natural (principal) grading. In addition, the projection $p_{2}$ induces a $\mathbb{C}[v]$-module structure on the coordinate ring $\mathbb{C}[\mathscr{L}]$, where $v$ denotes a coordinate function on $\mathbb{C}$.

By a result of Brion and the first author [2004, Theorem 1], the coordinate ring $\mathbb{C}[\mathscr{Z}]$ is isomorphic as a graded $\mathbb{C}$-algebra to the equivariant cohomology algebra $H_{T}^{*}(X)$. In fact, an isomorphism

$$
\rho: H_{T}^{*}(X) \rightarrow \mathbb{C}[\mathscr{E}]
$$

is defined as follows. Since the odd cohomology of $X$ is trivial (because $X^{T}$ is finite), the action $T: X$ is equivariantly formal, so the restriction map $i: H_{T}^{*}(X) \rightarrow$ $H_{T}^{*}\left(X^{T}\right)$ is injective. Note that

$$
H_{T}^{*}\left(X^{T}\right)=\bigoplus_{i=1}^{r} \mathbb{C}[v]_{i},
$$

where $v$ is an indeterminate and $\mathbb{C}[v]_{i}=H_{T}^{*}\left(\left\{x_{i}\right\}\right)$. Thus each $\alpha \in H_{T}^{*}(X)$ is determined by an $r$-tuple of polynomials $\left(\mathscr{A}_{1}, \ldots, \mathscr{A}_{r}\right)$ in $\mathbb{C}[v]$. Now if $(x, a) \in$ $\mathscr{Z}-(o, 0)$, then $x=\mu\left(a^{-1}\right) \cdot x_{j}$ for a unique index $j$, where $a \neq 0$. The restriction of $\alpha$ at $x_{j}$ is a polynomial function $\mathscr{A}_{j}(v)$. The isomorphism $\rho$ is defined by making $\rho(\alpha)$ the unique function on $\mathscr{E}$ defined by $\rho(\alpha)(x, v)=\mathscr{A}_{j}(v)$, if $x=\mu\left(a^{-1}\right) \cdot x_{j}$. 
Now note that $H_{T}^{*}(X)=\mathscr{H}_{T}^{0}(X)$. Furthermore, $\mu(1): X \rightarrow X$ defines an isomorphism $H_{T}^{*}(X) \cong H_{T_{1}}^{*}(X)$. Thus, we obtain a sequence of maps

$$
\mathbb{C}[\mathscr{L}] \stackrel{\rho^{-1}}{\longrightarrow} H_{T}^{*}(X) \stackrel{\mu(1)_{*}}{\longrightarrow} H_{T_{1}}^{*}(X) \stackrel{\Phi_{X}}{\longrightarrow} H^{0}\left(K_{X}^{*}\right)=\bigoplus_{X^{T_{1}}} \mathbb{C},
$$

where $K_{X}^{*}$ denotes the complex associated to the holomorphic vector field generated by the torus $T_{1}$. The composition $\Psi_{X}$ of these maps sends $F \in \mathbb{C}[\mathscr{E}]$ to the $r$-tuple $\rho^{-1}(F)(1)=\Phi_{X} \rho^{-1}(F)$, which, by Theorem 4.4, gives us the result.

\section{Examples}

The first example deals with a $\mathbb{G}_{m}$-action on $\mathbb{P}^{n}$ having two components of different dimensions.

Example 6.1. Let $X=\mathbb{P}^{n}$, and let $\mathbb{C}^{*}$ act on $X$ via

$$
t \cdot\left[a_{0}, a_{1}, \ldots, a_{n}\right]=\left[a_{0}, a_{1}, \ldots, t a_{n}\right] .
$$

Then $X^{T}=X_{1} \cup X_{2}$, where $X_{1}=\{[0,0, \ldots, 0,1]\}$, and $X_{2}=V\left(a_{n}\right) \cong \mathbb{P}^{n-1}$. Because $H_{T}^{p, q}(X)=0$ for $p \neq q$, we have $H_{T}^{p, p}(X)=H_{T}^{2 p}(X), \mathscr{H}_{T}^{s}(X)=0$ for $s \neq 0$, and $\mathscr{H}_{T}^{0}(X)=H_{T}^{*}(X)$. Similarly, $H_{T}^{*}\left(X^{T}\right)=\mathscr{H}_{T}^{0}\left(X^{T}\right)$. The image of $H_{T}^{*}(X)$ in $H_{T}^{*}\left(X^{T}\right)$ consists of all triples $(\alpha, \beta, \gamma)$ satisfying $\alpha \in H_{T}^{*}\left(X_{1}\right) \cong \mathbb{C}[t]$; $\beta \in H_{T}^{*}\left(X_{2}\right) \cong \mathbb{C}[t] \otimes H^{*}\left(X_{2}\right)$ with $\alpha(0)=\beta_{0}(0)$, where $\beta_{0}$ is the component of $\beta$ in $\mathbb{C}[t] \otimes H^{0}\left(X_{2}\right)$; and $\gamma=\sum c_{1}^{T}\left(E_{i}\right)$, where the $E_{i}$ are vector bundles on $X_{2}^{T}$. Recall from (5) that $c_{1}^{T}(E)=m t+c_{1}\left(E_{i}\right)$, where $t^{m}$ is the weight of the $\mathbb{G}_{m}$-action on the bundle on $X$ that restricts to $E_{i}$. The cochain map $\Phi_{X}$ sends $t \rightarrow 1$.

Example 6.2 (Toric varieties [Kaveh 2005]). Let $M=\left(\mathbb{C}^{*}\right)^{n}$, and let $X$ be a smooth projective $M$-toric variety. Let $\mathfrak{t}=\operatorname{Lie}(M)$ and $\mathfrak{t}_{\mathbb{R}} \subset \mathfrak{t}$ be the real vector space generated by the lattice of characters of $M$. Let $\gamma$ be a 1-parameter subgroup of $M$ in general position in the sense that the fixed point set $Z$ of the $\mathbb{C}^{*}$-action defined by $\gamma$ coincides with $X^{M}$. Hence $H^{p, q}(X)=0$ if $p \neq q$, so it follows that $\mathscr{H}^{0}(X)=H^{*}(X, \mathbb{C})$. Now let

$$
F_{0} \subset F_{1} \subset \cdots \subset F_{n}=H^{0}(Z, \mathbb{C})
$$

be the associated filtration. Finally, let $\Sigma$ be the fan of $X$ in $\mathfrak{t}_{\mathbb{R}}$. Each $z \in Z$ corresponds to a cone of maximal dimension $\sigma_{z}$ in $\Sigma$.

The equivariant cohomology $H_{T}^{*}(X, \mathbb{C})$, where $T=\left(S^{1}\right)^{n} \subset\left(\mathbb{C}^{*}\right)^{n}$, can be described as the algebra $\mathscr{A}$ of all continuous functions on $\mathfrak{t}_{\mathbb{R}}$ whose restriction to each cone of $\Sigma$ is given by a polynomial (conewise polynomial). Under this identification, $H_{T}^{2 i}(X, \mathbb{C})$ corresponds to the subspace $\mathscr{A}_{i}$ of $\mathscr{A}$ consisting of those functions whose restriction to each cone of maximal dimension is homogeneous of degree $i$. 
Let $Q$ denote the compact torus $\gamma\left(S^{1}\right)$. Then one can verify that the map $\Phi_{Z} \circ i_{Z}^{*}$ : $H_{Q}^{*}(X) \rightarrow H^{0}(Z, \mathbb{C})$ in Corollary 4.5 sends the restriction to $Q$ of a continuous conewise polynomial function $g$ to an element $\tilde{g}: Z \rightarrow \mathbb{C}$ defined by

$$
\tilde{g}(z)=g_{\mid \sigma_{z}}(\gamma) .
$$

It follows from Corollary 4.5 that $g \in \mathscr{A}_{i}$ if and only if $\tilde{g} \in F_{i}$. The fact that $F_{i} / F_{i-1} \cong H^{2 i}(X, \mathbb{C})$ was verified in [Kaveh 2005] using [Carrell and Lieberman 1977].

Example 6.3 (The flag variety $G / B$ ). Let $G$ be a connected semisimple group over $\mathbb{C}, B$ a Borel subgroup and $X=G / B$ the flag variety of $G$. Let $H$ be a maximal (algebraic) torus in $B$ and $\mathfrak{h}=\operatorname{Lie}(\mathrm{H})$. Recall that the fixed point set $X^{H}$ under left multiplication by $H$ is in one-to-one correspondence with the Weyl group $W=N_{G}(H) / H$ under the map $w=n H \rightarrow n B$. Since $H^{p, q}(X)=0$ for $p \neq q$, it follows that $H^{s}\left(K_{X}^{*}\right)=0$ if $s \neq 0$ for the holomorphic vector field induced by any one parameter subgroup of $H$. Now, $H_{H}^{*}(X, \mathbb{C})$ is isomorphic as a $\mathbb{C}$-algebra to $S \otimes_{S^{W}} S$ where $S^{W}$ denotes the subalgebra of $W$-invariants (see [Brion 1998, $§ 2$ Examples]).

We will first consider the regular case, which is well known but will be used in treating the general case.

(a) Suppose $h \in \mathfrak{h}$ induces a regular one parameter subgroup. That is, $Z=X^{H}$. Equivalently, the isotropy group $W_{h}$ of $h$ is trivial. Thus $H^{0}\left(K_{X}^{*}\right)=H^{0}(Z, \mathbb{C})=$ $\mathbb{C}^{W}$ under the identification $Z=W$. The map $H_{H}^{*}(G / B, \mathbb{C}) \rightarrow H^{0}(Z, \mathbb{C})$ obtained by localizing and setting $t=1$ is described as follows. Let $S=\mathbb{C}[\mathfrak{h}]$. Now, $H_{H}^{*}(X, \mathbb{C})$ is isomorphic as a $\mathbb{C}$-algebra to $S \otimes_{S^{W}} S$ where $S^{W}$ denotes the subalgebra of $W$-invariants (see [Brion 1998, $§ 2$ Examples]). Since $H^{*}(G / B, \mathbb{C}$ ) is generated by the Chern classes of line bundles, and such line bundles are always $H$-equivariant, we need only consider the image of an equivariant Chern class $c_{1}^{H}\left(L_{\lambda}\right)$, where $L_{\lambda}$ denotes the line bundle corresponding to a weight $\lambda \in \mathfrak{h}^{*}$. But it can be shown that $c_{1}^{H}\left(L_{\lambda}\right)=-\sum_{w \in W} 1 \otimes(w \cdot \lambda)$, and so $c_{1}^{H}\left(L_{\lambda}\right)$ is sent to the element $f_{\lambda} \in H^{0}(Z, \mathbb{C})$ defined by the condition

$$
f_{\lambda}(w)=-\langle w \cdot \lambda, h\rangle \text {. }
$$

This coincides with the representative of $c_{1}\left(L_{\lambda}\right)$ on $H^{0}(Z, \mathbb{C})$ calculated, for example, in [Carrell 1992]. The upshot is that $F_{1}$ is the image of $\mathfrak{h}^{*}$ under the quotient map $S \rightarrow \mathbb{C}[W \cdot h]$. This reproves the result that $H^{*}(X, \mathbb{C})=\mathrm{Gr} \mathbb{C}[W \cdot h]$, where the grading is taken with respect to the filtration obtained as the image of the filtration of $S$ associated to its natural grading. Note that $\mathbb{C}[W \cdot h]$ is the algebra of polynomials on the Weyl group orbit $W \cdot h$. 
(b) Suppose the element $h$ is nonregular. Let $\Phi$ be the root system of $(G, H)$ and $\Phi_{h}=\{\alpha \in \Phi \mid \alpha(h)=0\}$. Put

$$
\mathfrak{h}_{0}=\bigcap_{\alpha \in \Phi_{h}} \operatorname{ker} \alpha,
$$

and let $H_{0} \subset H$ be the corresponding torus. Finally, let $L$ denote the Levi subgroup $L=Z_{G}\left(H_{0}\right)$. For example, if $G=G L(n, \mathbb{C})$, and $H$ is the diagonal torus, put $h=$ $\operatorname{diag}\left(a_{1} I_{n_{1}}, a_{2} I_{n_{2}}, \ldots, a_{n_{r}} I_{n_{r}}\right)$, where $I_{l}$ is the $l \times l$ identity matrix, $n_{1}+\cdots+n_{r}=n$ and $a_{i} \neq a_{j}$ when $i \neq j$. Then $L=G L\left(n_{1}, \mathbb{C}\right) \times \cdots \times G L\left(n_{r}, \mathbb{C}\right)$. Then the Weyl group $W_{L}$ of $L$ is the isotropy subgroup of $h$ in $W$. Now $Z=X^{H_{0}}$ is a union of the flag varieties of $L$. More precisely, for $w \in W$, let $Z_{w}$ be the connected component of $Z$ containing $w B \in X^{H}$. One sees that each $Z_{w}$ is isomorphic to $L / L \cap B$ and $Z_{w}=Z_{w^{\prime}}$ for $w, w^{\prime}$ in the same right coset of $W_{L}$. Thus

$$
Z=\bigcup_{w \in W_{L} \backslash W} Z_{w}
$$

Hence $H^{*}(Z, \mathbb{C})=\bigoplus_{w \in W_{L} \backslash W} H^{*}(L / L \cap B)$. To obtain the filtration of $H^{*}(Z, \mathbb{C})$, take an element $t \in \mathfrak{h}$ that determines a regular 1-parameter subgroup of $H$. Let $\mathscr{L} \subset \mathfrak{h} \oplus \mathfrak{h}$ be the $W$-orbit of $(h, t)$, where $W$ acts diagonally on $\mathfrak{h} \oplus \mathfrak{h}$. One can write

$$
\mathscr{L}=\bigcup_{w \in W_{L} \backslash W} \mathscr{E}_{w},
$$

where $\mathscr{L}_{w}=\left\{\left(w^{-1} \cdot s, w^{-1} u^{-1} \cdot t\right) \mid u \in W_{L}\right\}$. The elements of $\mathscr{L}$ are in one-to-one correspondence with $X^{H}$, and each $\mathscr{E}_{w}$ corresponds to the $H$-fixed points in $Z_{w}$. Let $\mathbb{C}[\mathscr{E}]$ and $\mathbb{C}\left[\mathscr{L}_{w}\right]$ denote the coordinate rings of $\mathscr{L}$ and $\mathscr{L}_{w}$, respectively. From part (a), $H^{*}\left(Z_{w}\right) \cong \mathrm{Gr} \mathbb{C}\left[\mathscr{E}_{w}\right]$ for any $w \in W_{L} \backslash W$, where the filtration on $\mathbb{C}\left[\mathscr{E}_{w}\right]$ is induced by the degree. Hence

$$
H^{*}(Z, \mathbb{C}) \cong \bigoplus_{w \in W_{L} \backslash W} \operatorname{Gr} \mathbb{C}\left[\mathscr{E}_{w}\right]
$$

Put $\mathscr{A}=\bigoplus_{w \in W_{L} \backslash W} \operatorname{Gr} \mathbb{C}\left[\mathscr{E}_{w}\right]$. The following shows that the filtration on $\mathscr{A}$ is induced by the natural filtration on $\mathbb{C}[\mathscr{E}]$ given by the degree.

Proposition 6.4. An element $\left(f_{w}\right) \in \mathscr{A}$ lies in $F_{i}$ if and only if there exists an element $f \in \mathbb{C}[\mathscr{L}]$ with degree $\leq i$ and whose restriction to $\mathscr{L}_{w}$ is a representative for $f_{w}$ in $\mathrm{Gr} \mathbb{C}\left[\mathscr{L}_{w}\right]$.

Proof. Note that the result of part (a) implies that $H^{*}(X, \mathbb{C})$ is generated by $H^{2}(X, \mathbb{C})$. Hence the filtration is generated by $F_{1}$, that is, $F_{i}$ consists of all polynomials in the elements of $F_{1}$ of degree $\leq i$. Hence it is enough to verify the claim for $F_{1}$. Consider the line bundle $L_{\lambda}$ on $X$ corresponding to a dominant weight $\lambda$, 
and let $L_{\lambda, w}$ be the restriction of $L_{\lambda}$ to the small flag variety $Z_{w}$. Then, for each $w \in W_{L} \backslash W$, the weight of the action of $s$ on $L_{\lambda, w}$ is $\left\langle\lambda, w^{-1} \cdot s\right\rangle$. From (5),

$$
c_{1}^{s}\left(L_{\lambda, w}\right)=-\left\langle\lambda, w^{-1} \cdot s\right\rangle+c_{1}\left(L_{\lambda, w}\right),
$$

where $c_{1}^{s}$ denotes the equivariant Chern class for the $\mathbb{C}^{*}$-action induced by $s$. Then, from Theorem 4.4, (9) and (10) it follows that $c_{1}\left(L_{\lambda, w}\right)$ corresponds to the element $\left(f_{\lambda, w}\right)$ represented by the function

$$
\left(w^{-1} \cdot s, w^{-1} u^{-1} \cdot t\right) \mapsto-\left\langle\lambda, w^{-1} \cdot s\right\rangle-\left\langle\lambda, w^{-1} u^{-1} \cdot t\right\rangle .
$$

Now let $f_{\lambda}$ be the linear function on $\mathfrak{h} \oplus \mathfrak{h}$ given by $f(x, y)=-\lambda(x)-\lambda(y)$. From (11), the restriction of $f_{\lambda}$ to $\mathscr{L}_{w}$ gives a representative for $f_{w} \in \mathrm{Gr} \mathbb{C}\left[\mathscr{L}_{w}\right]$. The Proposition now follows because the $c_{1}\left(L_{\lambda}\right)$ span $H^{2}(X, \mathbb{C})$.

\section{References}

[Akyıldız 1982] E. Akyıldız, "Vector fields and cohomology of $G / P$ ", pp. 1-9 in Group actions and vector fields (Vancouver, 1981), edited by J. B. Carrell, Lecture Notes in Math. 956, Springer, Berlin, 1982. MR 84j:14054 Zbl 0498.14025

[Brion 1998] M. Brion, "Equivariant cohomology and equivariant intersection theory", pp. 1-37 in Representation theories and algebraic geometry (Montreal, PQ, 1997), NATO Adv. Sci. Inst. Ser. C Math. Phys. Sci. 514, Kluwer Acad. Publ., Dordrecht, 1998. Notes by Alvaro Rittatore. MR 99m:14005 Zbl 0946.14008

[Brion and Carrell 2004] M. Brion and J. B. Carrell, "The equivariant cohomology ring of regular varieties”, Michigan Math. J. 52:1 (2004), 189-203. MR 2005h:14112 Zbl 1084.14044

[Carrell 1992] J. B. Carrell, "Some remarks on regular Weyl group orbits and the cohomology of Schubert varieties", pp. 33-41 in Kazhdan-Lusztig theory and related topics (Chicago, IL, 1989), Contemp. Math. 139, Amer. Math. Soc., Providence, RI, 1992. MR 93k:14068 Zbl 0807.14040

[Carrell 1995] J. B. Carrell, "Deformation of the nilpotent zero scheme and the intersection ring of invariant subvarieties", J. Reine Angew. Math. 460 (1995), 37-54. MR 96e:14059 Zbl 0839.14008

[Carrell and Lieberman 1973] J. B. Carrell and D. I. Lieberman, "Holomorphic vector fields and Kaehler manifolds", Invent. Math. 21 (1973), 303-309. MR 48 \#4356 Zbl 0253.32017

[Carrell and Lieberman 1977] J. B. Carrell and D. I. Lieberman, "Vector fields and Chern numbers", Math. Ann. 225:3 (1977), 263-273. MR 55 \#8416 Zbl 0365.32020

[Carrell and Sommese 1979] J. B. Carrell and A. J. Sommese, "Some topological aspects of C* actions on compact Kaehler manifolds", Comment. Math. Helv. 54 (1979), 567-582. MR 80m:32032 Zbl 0466.32015

[Cartan 1951] H. Cartan, "La transgression dans un groupe de Lie et dans un espace fibré principal", pp. 57-71 in Colloque de topologie (espaces fibrés), Bruxelles, 1950, Georges Thone, Liège, 1951. MR 13,107f Zbl 0045.30701

[Feng 2003] H. Feng, "Holomorphic equivariant cohomology via a transversal holomorphic vector field”, Internat. J. Math. 14:5 (2003), 499-514. MR 2004j:32022 Zbl 1050.32013

[Frankel 1959] T. Frankel, "Fixed points and torsion on Kähler manifolds", Ann. of Math. (2) 70 (1959), 1-8. MR 24 \#A1730 Zbl 0088.38002 
[Fujiki 1979] A. Fujiki, "Fixed points of the actions on compact Kähler manifolds", Publ. Res. Inst. Math. Sci. 15:3 (1979), 797-826. MR 81c:32055 Zbl 0446.53021

[Ginzburg 1987] V. A. Ginzburg, "Equivariant cohomology and Kähler geometry", Funktsional. Anal. i Prilozhen. 21:4 (1987), 19-34. In Russian; translated in Funct. Anal. Appl. 21:4 (1987), 271-283. MR 89b:58013 Zbl 0656.53062

[Goresky et al. 1998] M. Goresky, R. Kottwitz, and R. MacPherson, "Equivariant cohomology, Koszul duality, and the localization theorem”, Invent. Math. 131:1 (1998), 25-83. MR 99c:55009 Zbl 0897.22009

[Kaveh 2005] K. Kaveh, "Vector fields and the cohomology ring of toric varieties", Canad. Math. Bull. 48:3 (2005), 414-427. MR 2007b:14111 Zbl 1094.14035

[Kirwan 1984] F. C. Kirwan, Cohomology of quotients in symplectic and algebraic geometry, Mathematical Notes 31, Princeton University Press, Princeton, NJ, 1984. MR 86i:58050 Zbl 0553.14020 [Lillywhite 2003] S. Lillywhite, "Formality in an equivariant setting", Trans. Amer. Math. Soc. 355:7 (2003), 2771-2793. MR 2004e:55016 Zbl 1021.55006

[Puppe 1974] V. Puppe, “On a conjecture of Bredon”, Manuscripta Math. 12 (1974), 11-16. MR 49 \#11502 Zbl 0277.57011

[Puppe 1979/80] V. Puppe, "Deformations of algebras and cohomology of fixed point sets", Manuscripta Math. 30:2 (1979/80), 119-136. MR 81e:57038 Zbl 0418.57015

[Teleman 2000] C. Teleman, "The quantization conjecture revisited", Ann. of Math. (2) 152:1 (2000), 1-43. MR 2002d:14073 Zbl 0980.53102

Received March 30, 2006. Revised October 20, 2006.

JIM CARRELL

DEPARTMENT OF MATHEMATICS

THE UNIVERSITY OF BRITISH COLUMBIA

Room 121, 1984 Mathematics RoAD

VANCOUVER, B.C.

CANADA V6T $1 \mathrm{Z2}$

carrell@math.ubc.ca

KiUmars KaVEH

DEPARTMENT OF MATHEMATICS

UNIVERSITY OF TORONTO

40 St. George StreEt

TORONTO, ONTARIO

CANADA M5S 2E4

kaveh@math.utoronto.ca

VOLKER PUPPE

UNIVERSITÄT KONSTANZ

FACHBEREICH MATHEMATIK UND STATISTIK

FACH D 197

D-78457 KONSTANZ

GERMANY

volker.puppe@uni-konstanz.de 